\title{
An Introduction to $\mathrm{Na}^{18} \mathrm{~F}$ Bone Scintigraphy: Basic Principles, Advanced Imaging Concepts, and Case Examples*
}

\author{
Robert L. Bridges, Chris R. Wiley, RT (R(CT)), John C. Christian, CNMT, and Adam P. Strohm, CNMT \\ Alaska Open Imaging Center, Anchorage, Alaska
}

\begin{abstract}
$\mathrm{Na}^{18} \mathrm{~F}$, an early bone scintigraphy agent, is poised to reenter mainstream clinical imaging with the present generations of stand-alone PET and PET/CT hybrid scanners. ${ }^{18} \mathrm{~F}$ PET scans promise improved imaging quality for both benign and malignant bone disease, with significantly improved sensitivity and specificity over conventional planar and SPECT bone scans. In this article, basic acquisition information will be presented along with examples of studies related to oncology, sports medicine, and general orthopedics. The use of image fusion of PET bone scans with CT and MRI will be demonstrated. The objectives of this article are to provide the reader with an understanding of the history of early bone scintigraphy in relation to $\mathrm{Na}^{18} \mathrm{~F}$ scanning, a familiarity with basic imaging techniques for PET bone scanning, an appreciation of the extent of disease processes that can be imaged with PET bone scanning, an appreciation for the added value of multimodality image fusion with bone disease, and a recognition of the potential role PET bone scanning may play in clinical imaging.
\end{abstract}

Key Words: multimodality fusion; $\mathrm{Na}^{18} \mathrm{~F}$; bone scintigraphy; PET/CT; bone; PET

J Nucl Med Technol 2007; 35:64-76

DOI: $10.2967 /$ jnmt.106.032870

\section{A} role of ${ }^{18} \mathrm{~F}-\mathrm{FDG}$ PET; the interest in new positron-emitting carbon-, nitrogen-, and fluorine-based radiopharmaceuticals; and the forward expansion into the exciting field of molecular imaging, an old veteran of the early days of bone scintigraphy impatiently waits off-stage ready to resume daily clinical work. ${ }^{18} \mathrm{~F}$ bone scintigraphy improves detection of bone disease, potentially improving diagnosis to levels commensurate with the improvement in diagnosis by ${ }^{18} \mathrm{~F}-\mathrm{FDG}$ PET in the field of oncology (1). In recent literature, the ${ }^{18} \mathrm{~F}$ bone scan has been reported to have improved sensitivity and specificity over conventional planar and

\footnotetext{
Received May 9, 2006; revision accepted Mar. 9, 2007.

For correspondence or reprints contact: Robert L. Bridges, MD, Alaska Open Imaging Center, 6911 DeBarr Rd., Anchorage, AK 99504.

E-mail: rbridgesmd@hotmail.com

*NOTE: FOR CE CREDIT, YOU CAN ACCESS THIS ACTIVITY THROUGH THE SNM WEB SITE (http://www.snm.org/ce_online) THROUGH JUNE 2009. COPYRIGHT ( 2007 by the Society of Nuclear Medicine, Inc.
}

SPECT bone scans (2). ${ }^{18} \mathrm{~F}$ bone scans are easy to perform and readily adaptable to general clinical imaging. This article will discuss some basic principles of imaging and also present advanced imaging using rigid fusion of ${ }^{18} \mathrm{~F}$ bone scans with CT and MRI.

\section{HISTORY}

The use of ${ }^{18} \mathrm{~F}$ for bone scintigraphy dates back to the early days of bone imaging in the 1950s and early 1960s. Initial attempts at bone scanning started with calcium and calcium analogs such as strontium. ${ }^{45} \mathrm{Ca}$, a $\beta$-emitter, required survey meter measurements of activity rather than imaging. With the rectilinear scanner developed by Benedict Cassen in 1951 (3), ${ }^{85} \mathrm{Sr}$ (513 keV; half-life, $65 \mathrm{~d}$ ) could be imaged 2-7 d after injection (4). However, because of its long half-life and energies, this isotope was limited to patients with known metastatic disease. ${ }^{87 \mathrm{~m}} \mathrm{Sr}$ (338 keV; half-life, $2.8 \mathrm{~h}$ ) provided a better energy but very short half-life (4). At that juncture, $\mathrm{Na}^{18} \mathrm{~F}(511 \mathrm{keV}$; halflife, $110 \mathrm{~min}$ ) was introduced in the early 1960s but required ready accessibility to a production site (4). ${ }^{18} \mathrm{~F}$ could be imaged with the general-purpose rectilinear scanner and dedicated positron detectors as early as 1963. For historical perspective, initial development of the coincidence positron detector dates back to 1951 with the work of Drs. William Sweet and Gordon Brownell in the collaboration of Massachusetts General Hospital and Massachusetts Institute of Technology in 1951 (5).

Three important events led to the abandonment of the early bone-scanning isotopes. The development of the ${ }^{99} \mathrm{Mo} /{ }^{99 m} \mathrm{Tc}$ generator in 1958 at Brookhaven Laboratories by Tucker and Greene, and further refined with the collaboration of Powell Richards, opened the door to bone imaging (6). In 1971, Subramanian and McAfee developed for bone imaging the initial ${ }^{99 \mathrm{~m}} \mathrm{Tc}$-phosphate compounds, which were optimal in energy for the Anger camera (7). The third event was the introduction of the $\gamma$-camera, first the pinhole camera in 1952 and then the scintillation camera in 1957 (8). The $\gamma$-camera could image with ${ }^{18} \mathrm{~F}$, but poorly, because it performed best at lower energy levels.

In 1972, the Food and Drug Administration approved ${ }^{18} \mathrm{~F}$ for bone scintigraphy. In 2000, as part of the modernization 
of the Food and Drug Administration and the handling of new drug applications, ${ }^{18} \mathrm{~F},{ }^{13} \mathrm{~N}$, and ${ }^{18} \mathrm{~F}-\mathrm{FDG}$ were approved for PET in the areas of bone scintigraphy, cardiac perfusion, and oncology/neurology, respectively (9).

The "rediscovery" of ${ }^{18} \mathrm{~F}$ bone scintigraphy has resulted in a trickle of studies and papers. Whole-body skeletal imaging was discussed in articles in 1993, yielding limited but encouraging results dealing with metabolic disease and malignancy $(10,11)$. Schirrmeister found that PET bone scanning elevated the sensitivity and specificity of bone scanning from levels of about $80 \%$ to nearly $100 \%$ (1). A recent Israeli study further confirmed the advantages of PET bone scanning, focusing on the PET/CT hybrid scanner in comparisons with planar imaging and SPECT (2).

\section{PHYSICS AND PHARMACOKINETICS OF ${ }^{18} \mathrm{~F}$}

${ }^{18} \mathrm{~F}$ is a positron emitter with a half-life of $109.7 \mathrm{~min}$. It has an unstable nucleus because of an excess of protons. Nuclear stability is achieved via one of two pathways that reduce the number of protons in the nucleus through conversion of one of the protons to a neutron. Three percent of decay is by electron capture, where an electron from an inner energy level of the atom may be captured by the nucleus, with subsequent emission of characteristic x-rays of oxygen as a higher-orbit electron drops into the lower-orbit void of the newly transformed atom. Ninety-seven percent of decay is by emission of a positron having a maximum energy of $0.635 \mathrm{MeV}$. The positron cannot immediately interact with an electron and must decelerate (thermalize) to nearly resting energy before annihilation with an electron can occur (12). This deceleration distance from emission of the positron to annihilation is less than $2 \mathrm{~mm}(13,14)$. Because the positron does not have to fully decelerate to resting mass to interact with an electron, its energy at the time of annihilation can be $511 \mathrm{keV} \pm 40 \mathrm{eV}$, with the resulting 2 antiparallel photons separating at $180^{\circ}$ with $\pm 0.25^{\circ}$ of angular spread. The photons have a half-value layer of $4.1 \mathrm{~mm}$ of lead, compared with $0.17 \mathrm{~mm}$ for ${ }^{99 \mathrm{~m}} \mathrm{Tc}$ (12).

${ }^{18} \mathrm{~F}$ can be produced by deuteron bombardment of ${ }^{20} \mathrm{Ne}$ or proton bombardment of ${ }^{18} \mathrm{O}$. The latter method is used in present-day commercial cyclotrons. $\mathrm{Na}^{18} \mathrm{~F}$ has a molecular weight of 40.99 (12). $\mathrm{Na}^{18} \mathrm{~F}$, created in the cyclotron cell, is the chemical ${ }^{18} \mathrm{~F}$ precursor used to commercially make ${ }^{18} \mathrm{~F}$ FDG and, as such, is readily available.

After intravenous injection, the ${ }^{18} \mathrm{~F}$ ions rapidly equilibrate, principally within the extracellular fluid space. Rapid clearance is to bone and excretion into the urine. Greater than $20 \%$ of the injected dose is cleared through the kidneys in the first $1-2 \mathrm{~h}$. Fluorine deposition favors the axial (spine and pelvis) over the appendicular (shoulder girdles and limbs) skeleton and is greater for joints than for shafts of long bones (12). As with ${ }^{99 \mathrm{~m}}$ Tc-based bone agents, which adhere to bone by chemical absorption, fluorine is directly incorporated into the bone matrix, converting hydroxyapatite to fluoroapatite. Fluorine is incorporated at a rate twice that of the phosphonate mechanism. Unlike a portion of injected ${ }^{99 \mathrm{~m}} \mathrm{Tc},{ }^{18} \mathrm{~F}$ is not protein-bound $(15,16)$.

The target and critical organs are both bone. The estimated radiation dose in adult humans is $0.0120 \mathrm{mGy} / \mathrm{MBq}$ for the bone surfaces, compared with $0.035 \mathrm{mGy} / \mathrm{MBq}$ for 99m Tc-methylene diphosphonate (MDP). The bladder wall radiation dose is $0.19 \mathrm{mGy} / \mathrm{MBq}$, versus $0.03 \mathrm{mGy} / \mathrm{MBq}$ for ${ }^{99 \mathrm{~m}}$ Tc-MDP $(12,17)$.

Because of the high rate of clearance by bone and renal excretion, and the small amount of renal activity that normally persists, the ${ }^{18} \mathrm{~F}$ "superscan" may be difficult to interpret.

\section{METHODS AND SCANNERS}

The state of the art for PET is the PET/CT hybrid scanner. As noted in the recent literature, the PET/CT scanner can perform PET bone scans, providing both metabolic and anatomic information in near-anatomic alignment. The quoted point-source resolution for the hybrids varies from the sub-5-mm to sub-7-mm range $(13,18,19)$. Stand-alone PET scanners characteristically have a sub-5-mm pointsource resolution (13). The practical resolution for clinical imaging with modern PET systems is around $8 \mathrm{~mm}$. Conventional SPECT with $99 \mathrm{mTc}-\mathrm{MDP}$ has a resolution of around $15 \mathrm{~mm}$. The latest SPECT/CT scanners can have a quoted point-source resolution in the 4-mm range. Thus, SPECT/CT may have greater resolution and still use standard available isotopes. However, SPECT/CT will not be as fast as PET, nor can it cover the entire body in a single session. "Stitching" software would be required for combining the multiple SPECT acquisitions.

For this article, PET was performed on a CPET + scanner (Philips), which is a 3-dimensional (3D) curved-detector $\mathrm{NaI}$ system with a point-source resolution of $4.9 \mathrm{~mm}$. Scanning was performed in a routine fashion for this scanner, with the injected dose calculated for the patient's weight. Although $175 \mathrm{MBq}$ can be considered a comfortable maximum dose for the CPET + , pixel overflow occurred in 1 case in an especially hot lesion. This problem should not occur with the other PET scanner crystals.

For bismuth germanate, lutetium oxyorthosilicate, germanium oxyorthosilicate, or the new yttrium lutetium oxyorthosilicate crystal PET scanners, an injected dose of $370 \mathrm{MBq}$ is recommended. Imaging time per table position should be the same as for ${ }^{18}$ F-FDG PET, although modification with experience is recommended.

Should blood-pool images be required, immediate imaging is mandatory. ${ }^{18} \mathrm{~F}$ is so rapidly cleared that the bloodpool phase is very short. Delayed imaging occurs $1 \mathrm{~h}$ after injection. We recommend extra hydration after injection to facilitate clearance of residual tracer, and we recommend timely voiding to lessen the dose to the bladder wall. Walking or similar physical activity after injection also appears to help reduce background activity. Depending on the scanner, imaging time may be longer or shorter than 
planar bone scans but is always significantly shorter than SPECT scans for the areas scanned.

CT scans were performed on an Aquilion 16-channel scanner (Toshiba). For bone scintigraphy, CT scans were performed with 1-mm-thick slices through the area of interest, be it limited or the whole body.

MRI was performed on an open scanner-either the 0.3-T Airis-II (Hitachi) or the 0.35-T OpArt (Toshiba) - at the centers or with outside MRI studies, several on 1.5-T systems. Suitable MR image sequences, such as short- $\tau$ inversion recovery for contrast or T1-weighted for architectural detail, are helpful. Most MRI is performed with the patient quietly breathing, producing a breathing pattern and positioning of the diaphragm and adjacent organs similar to those during the PET acquisition to aid in software fusion of PET and MRI sequences. Furthermore, the improved soft-tissue characterization of MRI musculoskeletal imaging lends itself to multimodality fusion. The individual MRI sequences in transaxial, sagittal, coronal, and oblique planes can be individually fused, maintaining optimal MRI resolution.

Software fusion was performed on a commercially available fusion workstation, the Fusion 7D (Siemens). All fusions were rigid and did not use the deformable fusion, approved by the Food and Drug Administration, available on the workstation. The initial fusion was automatic weighted mutual information. Final adjustment was manual, if needed. Rigid fusion was used to provide imaging that would be representative of any workstation capable of rigid fusion, whether commercial, shareware, or freeware. Landmarks are readily identifiable between the PET bone scan images and the CT or MRI scan because of the high-contrast resolution afforded by PET bone scintigraphy. Patients were positioned to best replicate the same body alignment on the different scanners. The CT studies were

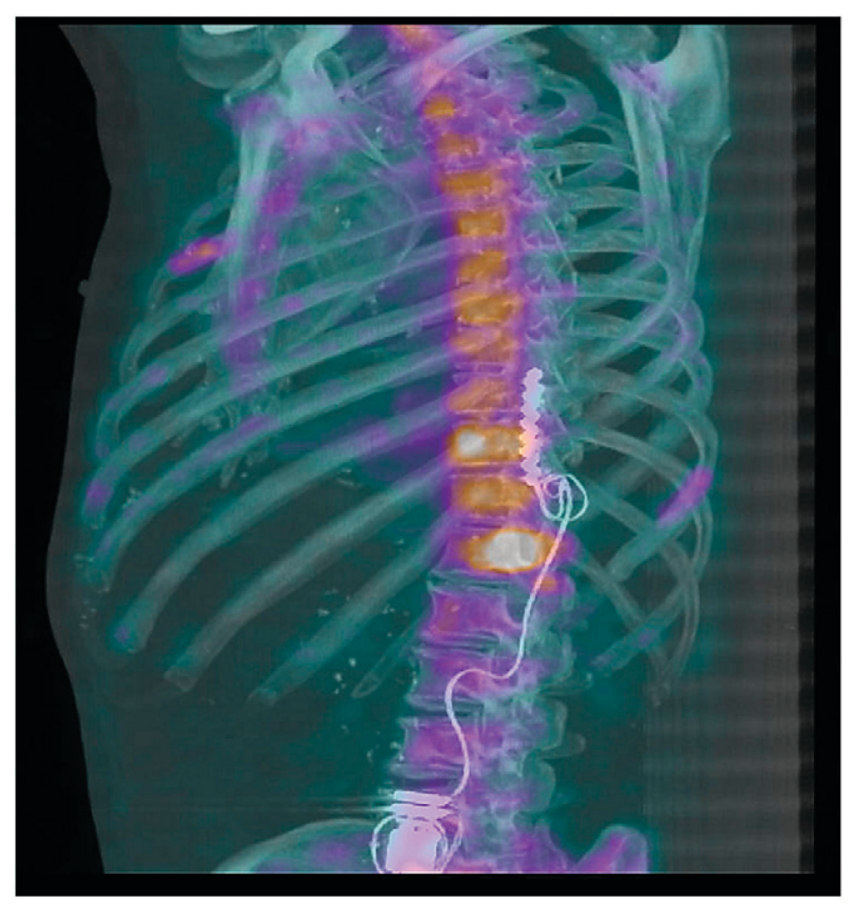

FIGURE 1. $3 \mathrm{D}{ }^{18} \mathrm{~F}-\mathrm{FDG}$ PET/CT fused image showing rib and vertebral metastases. Electronic pain control module and electrodes are present.

obtained at end-expiratory breath-hold where tolerated or during shallow free breathing, as needed. A representative obliquely oriented maximum-intensity projection (MIP) of a CT and PET bone fusion image of vertebral body and rib metastases is shown in Figure 1.

The MIP images in Figure 2 are characteristic examples of non-attenuation-corrected emission and attenuation-corrected images for a small-framed patient and an obese patient. There
FIGURE 2. ${ }^{18} \mathrm{~F}$ bone scan MIP images without and with attenuation correction for small and very large patients. (A and B) Emission-only and attenuationcorrected anterior view of small-framed patient. (C and D) Emission-only and attenuation-corrected anterior view of obese patient. Attenuation correction is vital for detection of small lesions and provides excellent imaging of very large patients. PET is more sensitive than single-photon imaging to attenuation effects.
A

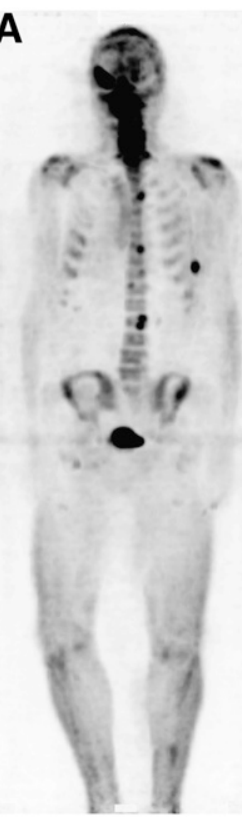

B

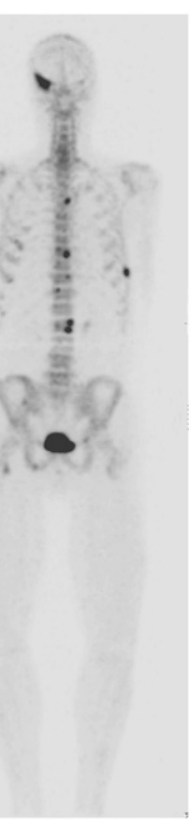

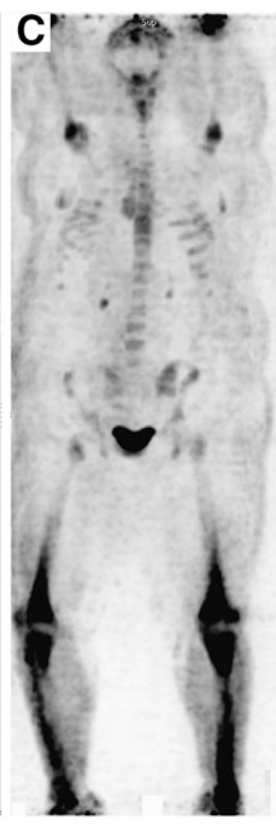

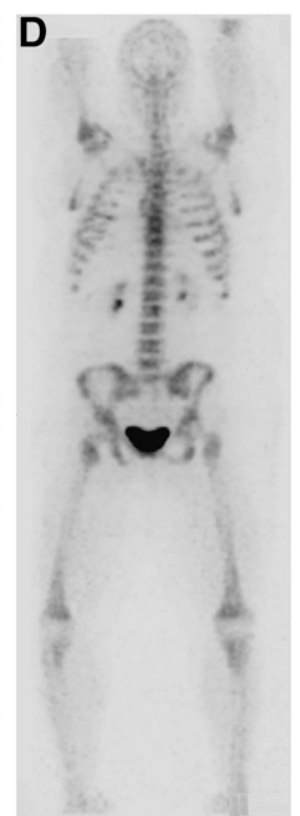



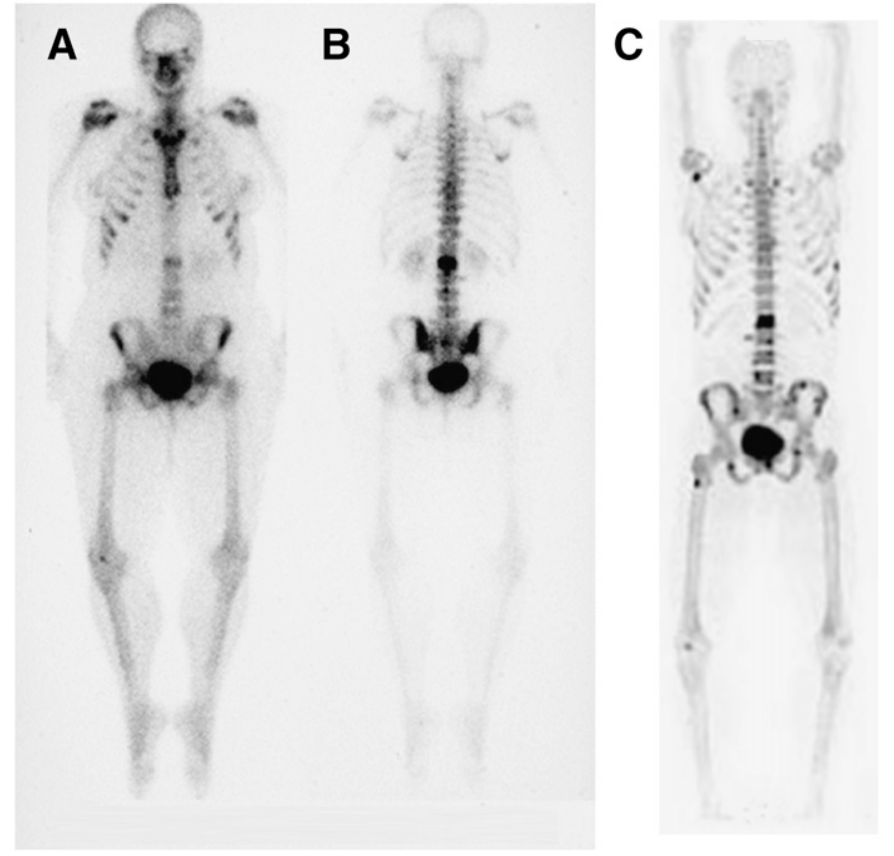

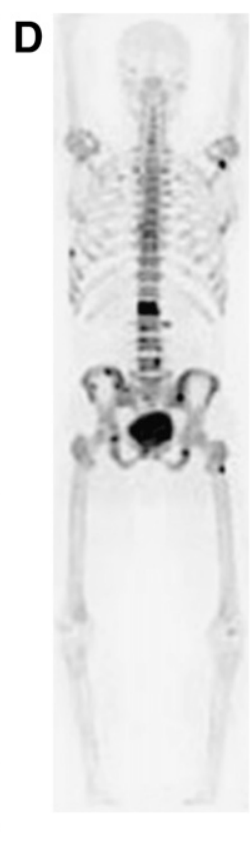

FIGURE 3. Standard 99mTc-MDP planar bone scan and ${ }^{18} \mathrm{~F}$ bone scan of same patient obtained within a few days of each other (courtesy of Seattle Nuclear). (A and B) Anterior and posterior views showing increase in uptake in vertebra and left rib. (C and $D$ ) Anterior and posterior MIP views showing numerous additional metastases. Even in retrospect, few of the ${ }^{18} \mathrm{~F}$ lesions can be seen on planar images. may be a temptation to shorten the PET scan to nonattenuation images, but the loss of image quality and information was found early on to be too great. Very little residual softtissue background was present after attenuation correction. The kidneys were only faintly visible on the attenuationcorrected scan. Small lesions might not be seen on standard 99m Tc-MDP imaging but easily seen on PET bone scanning. Unlike single-photon imaging without correction, the transmission correction and the higher energy of the positrons tend to mitigate some of the effects of traversing longer tissue distances from skeleton to detectors. The new time-of-flight PET scanners will actually perform better with larger patients because of improved spatial localization of the annihilation events.

A side-by-side comparison of standard planar ${ }^{99 \mathrm{~m}} \mathrm{Tc}-$ MDP and PET bone scans of a breast cancer patient is shown in Figure 3 (courtesy of Seattle Nuclear). The scans were obtained within days of each other specifically to demonstrate the differences between standard planar and PET bone imaging. Although the planar scan appeared to show focal upper lumbar and possibly rib disease, the PET bone scan clearly delineated numerous additional sites of metastatic disease, particularly very small lesions in the pelvic area.

Several articles have shown the ability of ${ }^{18}$ F-FDG PET to identify malignant bone involvement. Malignancy initially replacing marrow may not evoke an osteoblastic response with conventional bone scintigraphy $(20) .{ }^{18} \mathrm{~F}$ FDG PET is superior for detecting the soft-tissue tumor component creating aggressive lytic lesions but may fail to adequately detect small osteoblastic lesions that may be seen in prostate and breast cancer. The osteoblastic

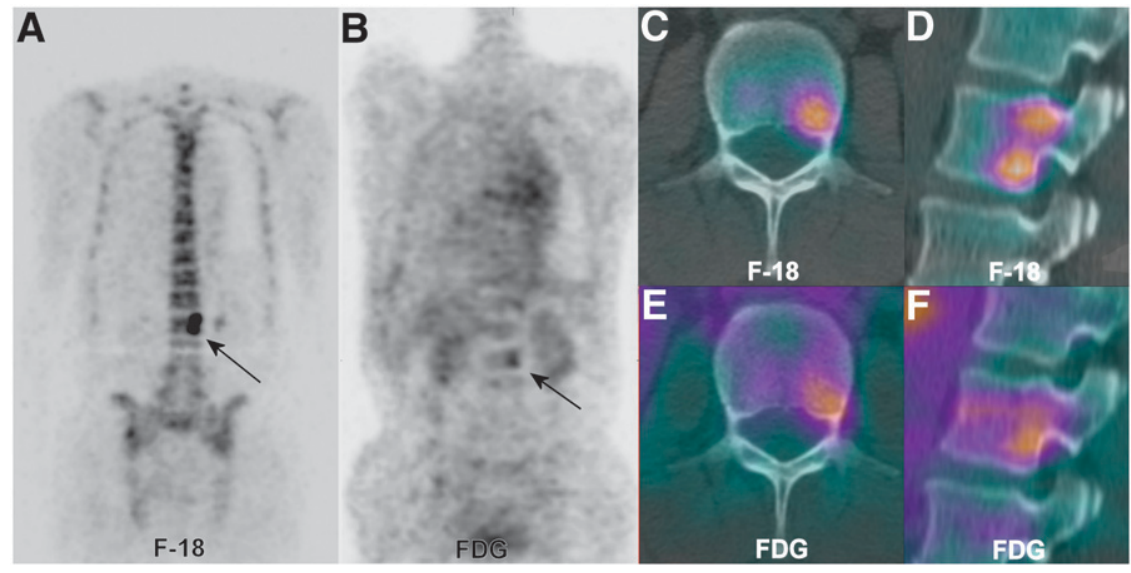

FIGURE 4. ${ }^{18} \mathrm{~F}-\mathrm{FDG}$ PET and companion ${ }^{18} \mathrm{~F}$ bone scan of metastatic disease of lumbar vertebrae. ${ }^{18} \mathrm{~F}$ bone scan, ${ }^{18} \mathrm{~F}-$ FDG PET scan, and CT study are from same patient taken separately in same week. (A) Coronal slice from ${ }^{18} \mathrm{~F}$ bone scan showing 2 distinct lesions in left portion of $\mathrm{L} 2$ vertebral body (arrow). (B) Identical slice from ${ }^{18} \mathrm{~F}-\mathrm{FDG}$ PET scan faintly positive for more inferior area of $L 2$ (arrow). (C and D) Transaxial and sagittal fused $\mathrm{CT}$ and ${ }^{18} \mathrm{~F}$ bone scans showing inferior lesion on transaxial slice and 2 lesions on reformatted sagittal slice through same area. ( $\mathrm{E}$ and F) ${ }^{18} \mathrm{~F}-\mathrm{FDG}$ PET fusion with same CT scan shows only inferior lesion in L2. Physiologic osteoblastic uptake in tumor may be severalfold greater with ${ }^{18} \mathrm{~F}$ than with ${ }^{18} \mathrm{~F}-\mathrm{FDG}$ or ${ }^{99 \mathrm{~m} T c-M D P}$. 
response of bone to disease may be severalfold greater than the 3-5 times greater uptake in tumor tissue versus benign soft tissues for ${ }^{18} \mathrm{~F}-\mathrm{FDG} .{ }^{18} \mathrm{~F}$ has been reported to be more sensitive than ${ }^{99 \mathrm{~m} T c-M D P}$ for detection of lytic lesions (15). A recent case report suggests that the flare phenomenon from healing bone is more sensitively seen with ${ }^{18} \mathrm{~F}$ than with ${ }^{99 \mathrm{~m}} \mathrm{Tc}-\mathrm{MDP}(21)$. The report described a case in which therapy resulted in a vertebral body flare seen with ${ }^{18} \mathrm{~F}$ but not with ${ }^{99 \mathrm{~m}} \mathrm{Tc}-\mathrm{MDP}$.

The bone scan seen in Figure 4 is of a patient who underwent ${ }^{18} \mathrm{~F}$-FDG PET for staging of a poorly differentiated adenocarcinoma of unknown primary, followed less than 1 wk later by the ${ }^{18} \mathrm{~F}$ PET bone scan. The same representative coronal slice for the bone scan shows 2 adjacent areas of intense uptake in the left portion of the lumbar vertebrae, whereas a proportionately less prominent uptake with ${ }^{18} \mathrm{~F}$-FDG is noted for the inferior aspect of the vertebrae. The fused $\mathrm{CT}$ and PET bone scan identifies 2 lesions, whereas ${ }^{18} \mathrm{~F}-\mathrm{FDG}$ PET/CT detected only the more inferior of the 2 metastases. Numerous other lesions present on the bone scan, such as in the pelvis, are also below the threshold of detection for ${ }^{18} \mathrm{~F}-\mathrm{FDG}$ PET.

Figure 5 is from a dual-tracer-combined ${ }^{18} \mathrm{~F}-\mathrm{FDG}$ and ${ }^{18} \mathrm{~F}$ - acquisition. The patient had breast cancer, and the scan was performed to assess the extent of disease. This dual tracer uses a 10:1 ${ }^{18} \mathrm{~F}-\mathrm{FDG}:{ }^{18} \mathrm{~F}$ injected dose to simultaneously detect both soft-tissue and osseous (lytic and sclerotic) involvement with just 1 scan. For appropriate standardized uptake values of soft-tissue lesions on the scan, the standardized uptake value is calculated using only the ${ }^{18} \mathrm{~F}$-FDG dose, not using the total injected dose of the 2 tracers. For these images, in addition to extensive softtissue disease including right axillary lymph nodes and the liver, a distinct bone metastasis can be seen in the S2 vertebral body coinciding with a sclerotic focus in the bone on PET/CT fusion. The role of the dual-tracer scan may be as a survey tool to assess the full extent of disease in 1 scan while reducing the cost and imaging time for the patient.

\section{CASE STUDIES}

The following case studies highlight our experience with ${ }^{18} \mathrm{~F}$ PET bone scanning and correlative fusion imaging.

\section{Case A}

Case A, shown in Figure 6, was a 56-y-old woman with metastatic colon cancer who was imaged with complementary ${ }^{18} \mathrm{~F}-\mathrm{FDG}$ and ${ }^{18} \mathrm{~F}$ PET. As with the ${ }^{99 \mathrm{~m} T c-}$ phosphonate tracers, ${ }^{18} \mathrm{~F}$ will have increased uptake in areas of necrotic tissue and tumor calcifications. In this case, both scans were ordered to stage the cancer with the hope of a potential course of multidisciplinary curative therapy. The montage of images in Figure 6 compares the appearance of ${ }^{18} \mathrm{~F}$ with $\mathrm{CT}$ and ${ }^{18} \mathrm{~F}$-FDG PET. This patient presented with rectal cancer metastases to an upper pelvic lymph node and large lesions confined to the right lobe of the liver. The companion ${ }^{18} \mathrm{~F}$ bone scan was negative for

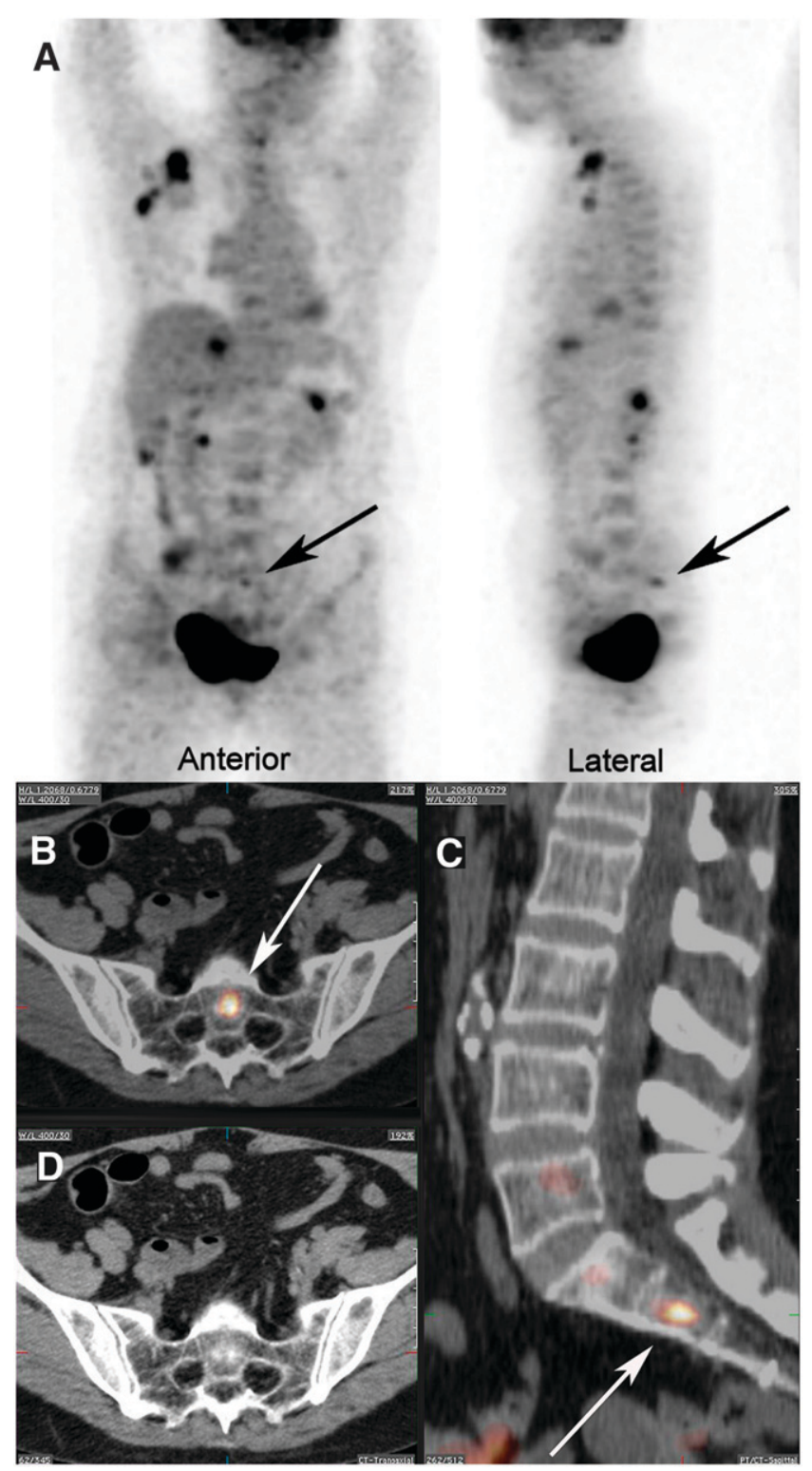

FIGURE 5. Dual-tracer acquisition: combined injection of ${ }^{18} \mathrm{~F}-\mathrm{FDG}$ and ${ }^{18} \mathrm{~F}$. (A) Two views of combined tracer showing soft-tissue, lytic bone, and osteoblastic bone lesions. Arrows point to small osteoblastic lesion in sacrum. (B and C) Fused $\mathrm{CT}$ and ${ }^{18} \mathrm{~F}-\mathrm{FDG} /{ }^{18} \mathrm{~F}$ PET characterizing lesion (arrows) as small and osteoblastic in central body of S2. (D) CT image of $B$ with fusion shows sclerotic nature of S2 lesion. Note sclerotic focus centrally within S2 body in lower left corner of CT image and same area on fused images. This area would probably not have been visualized on plain ${ }^{18} \mathrm{~F}-\mathrm{FDG}$ scan.

bony metastases but had faint activity in the right upper quadrant. CT through the liver showed a large, ill-defined mass in the right lobe of the liver, permeative in pattern and with stippling of calcifications. The ${ }^{18} \mathrm{~F}-\mathrm{FDG}$ PET/CT fusion image showed a prominent hypermetabolic rim with a photopenic central area from probable hypoxic and necrotic tissue. ${ }^{18} \mathrm{~F}$ and $\mathrm{CT}$ fusion delineated the 


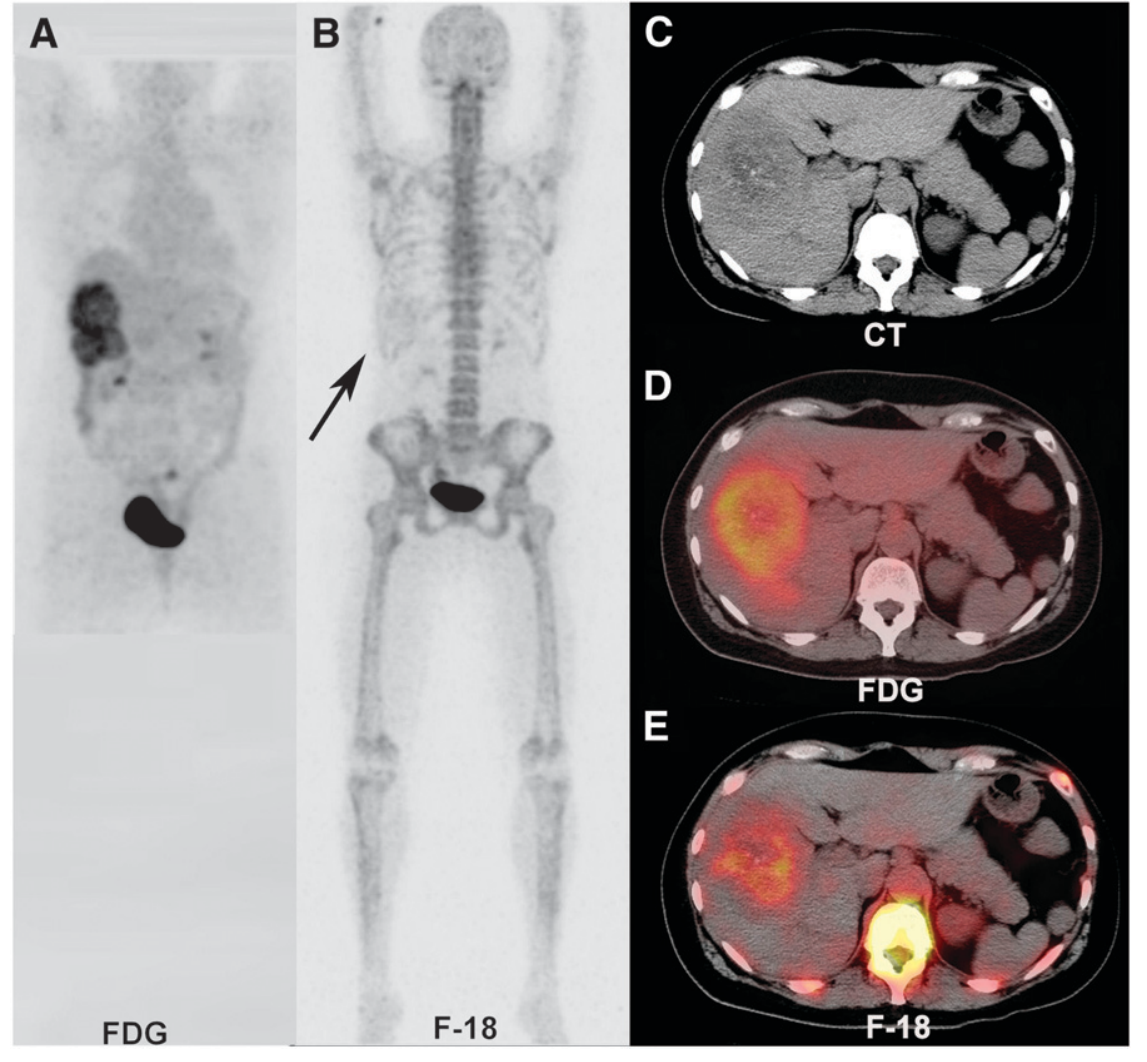

FIGURE 6. ${ }^{18} \mathrm{~F}-\mathrm{FDG}$ and ${ }^{18} \mathrm{~F}$ scans of metastatic colon cancer fused to same "isoaligned" CT scan. (A) Large lesions dominating right lobe of liver. (B) Faint tracer uptake (arrow) in right upper quadrant in otherwise negative PET bone scan. (C) Transaxial CT slice of larger lesion showing calcifications in tumor. (D) Prospective fusion of ${ }^{18} \mathrm{~F}-\mathrm{FDG}$ PET and CT showing peripheral hypermetabolic margin of tumor with decreased metabolism in center of mass. (E) Retrospective fusion of ${ }^{18} \mathrm{~F}-\mathrm{FDG}$ PET and previous CT delineating extent of tumor calcifications and central necrosis. uptake in the ${ }^{18} \mathrm{~F}$-FDG-photopenic area within the tumor. Both ${ }^{18} \mathrm{~F}-\mathrm{FDG}$ and ${ }^{18} \mathrm{~F}$ showed central photopenic areas probably reflecting poorly perfused or frankly necrotic tumor.

\section{Case B}

Case B, shown in Figure 7, was a 24-y-old female powerrunner who presented with knee pain. She initially underwent an ${ }^{18} \mathrm{~F}$ bone scan that showed a circumferential area of
A

B

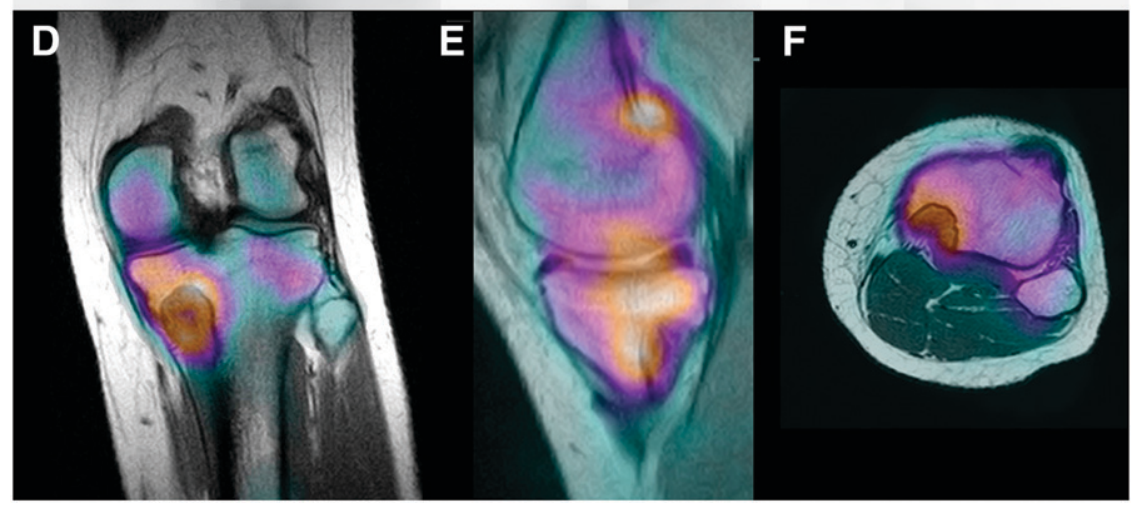

C

FIGURE 7. Medial tibial lesion in runner: positive ${ }^{18} \mathrm{~F}$ scan followed by MRI. (A-C) Coronal, sagittal, and axial images of main lesion and uptake in plateau, adjacent medial femoral condyle, and gastrocnemius origin. (D-F) Complementary images from fusion with MRI clarify extent and distribution of uptake. Principal uptake is due to weakened plateau from underlying benign tumor. 


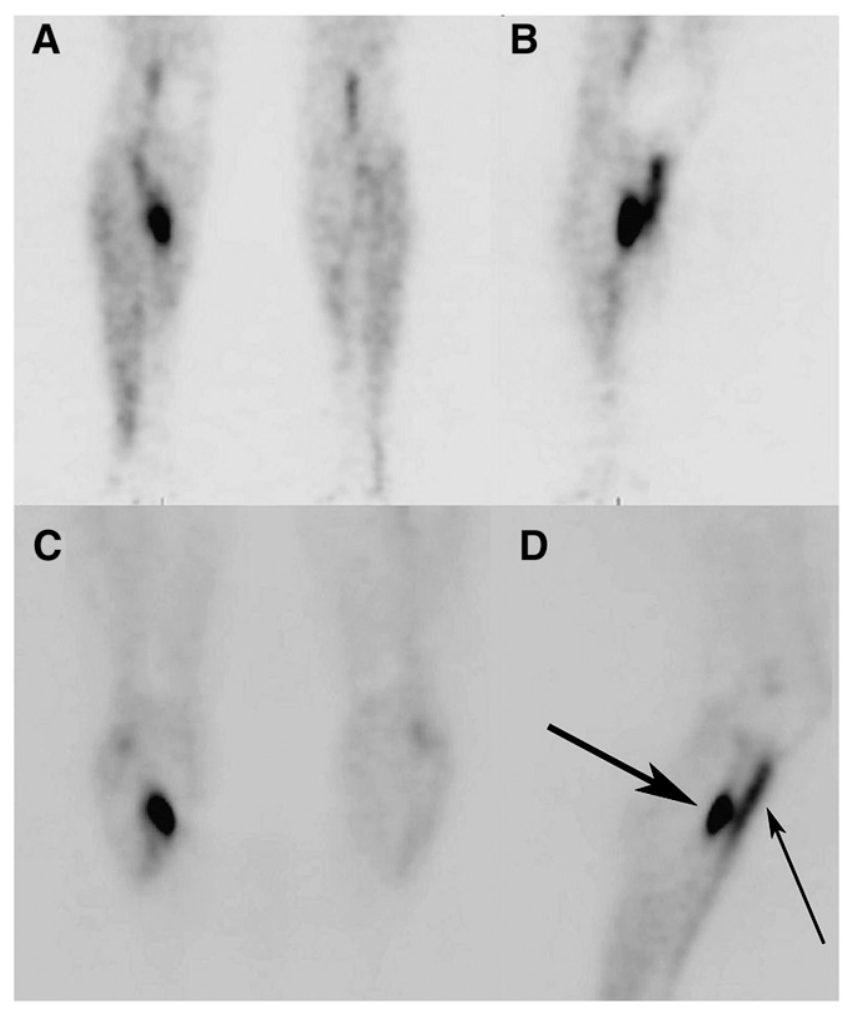

FIGURE 8. Stress fracture and shin splints of lower leg. (A and B) Blood-pool scan showing posterior stress fracture and anterior shin splints. (C and D) Delayed-image scan of stress fracture (large arrow) and shin splints (small arrow).

increased activity with central photopenia localizing to the medial tibia plateau area. A focal increase in uptake was present in the medial femoral condyle and the medial head of the gastrocnemius muscle origin. MRI detected a well- circumscribed benign-appearing "bubbly" lesion in the peripheral posterior-medial metaphyseal aspect of the tibia. Fusion of the studies showed some activity within the lesion but accentuated activity at the lesion margins, especially the weight-bearing margins. This preferential localization to the weight-bearing margin suggested that the pain was secondary to weakening of the plateau by the underlying benign lesion in this athlete.

\section{Case C}

Case C, shown in Figure 8, was a young female athlete with leg pain and normal findings on radiography. As with case B, PET bone scanning can not only replicate but also improve on general bone studies. A classic stress fracture of the tibia was seen, with accompanying "shin splints" as imaged with the PET scanner. The blood-pool phase and delayed images identified the size, location, and extent of these stress fractures. The quantitative uptake values can be compared with future scans to assess an objective increase or decrease in activity, rather than the relative values of standard planar and SPECT scans.

\section{Case D}

Case D, shown in Figure 9, was a young female athlete with back pain. She experienced back pain only during strenuous competition; the pain lessened or resolved between sporting events. The MRI study revealed no disk disease but found a linear area of decreased signal in the pedicle-pars interarticularis area surrounded increased signal on T2-weighted sagittal and the transaxial images. Such increased signal on T2-weighted imaging can represent bone edema, inflammation, or infection. The PET bone scan confirmed the presence of a stress fracture (an incipient pars

FIGURE 9. Incipient fracture of right L5 pedicle/pars interarticularis area in young athlete. (A and E) Sagittal and transaxial T2-weighted MR images of incipient pars fracture. Arrows point to incomplete linear defect within, surrounded by edema. $(B, C$, and $F)$ Bone scan localizing and confirming lesion in right $\mathrm{L} 5$ pars interarticularis area (arrows). (D) MIP of emission-only scan of patient. Note sharply defined lesion not obscured by bone edema.
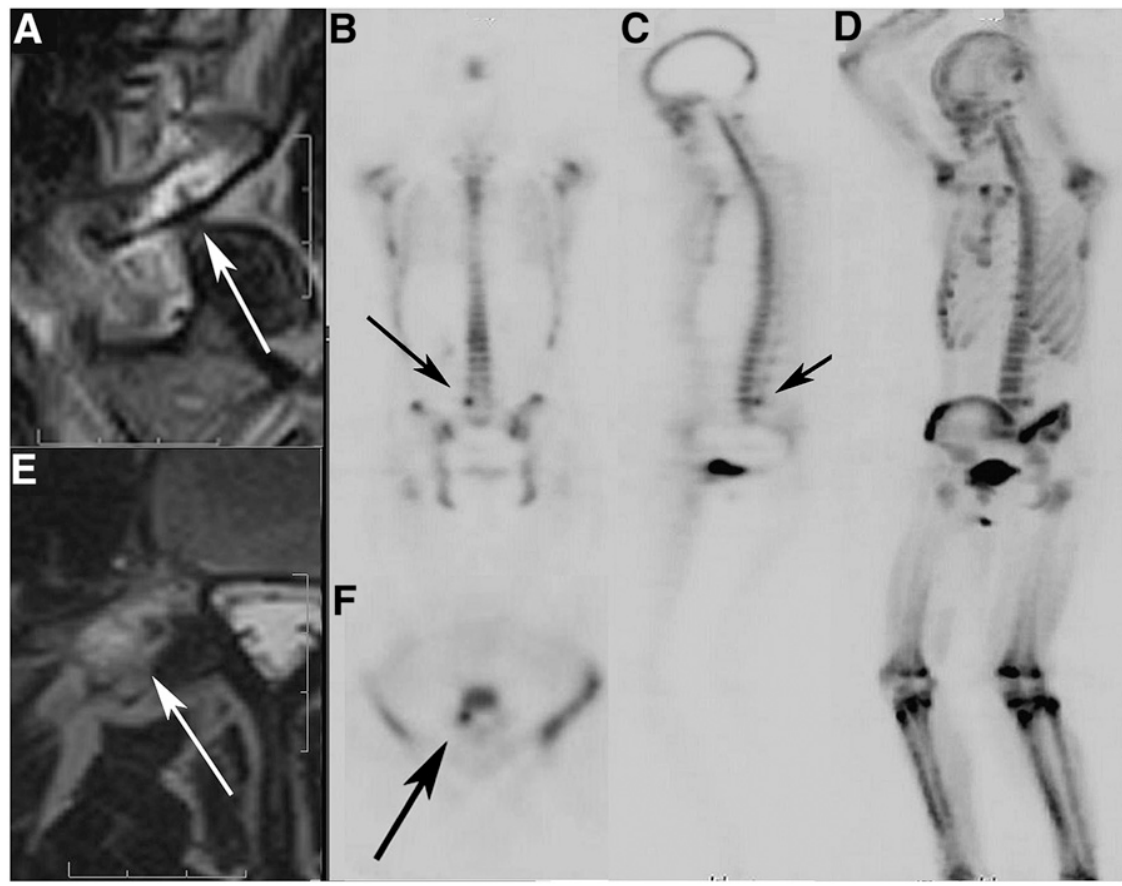

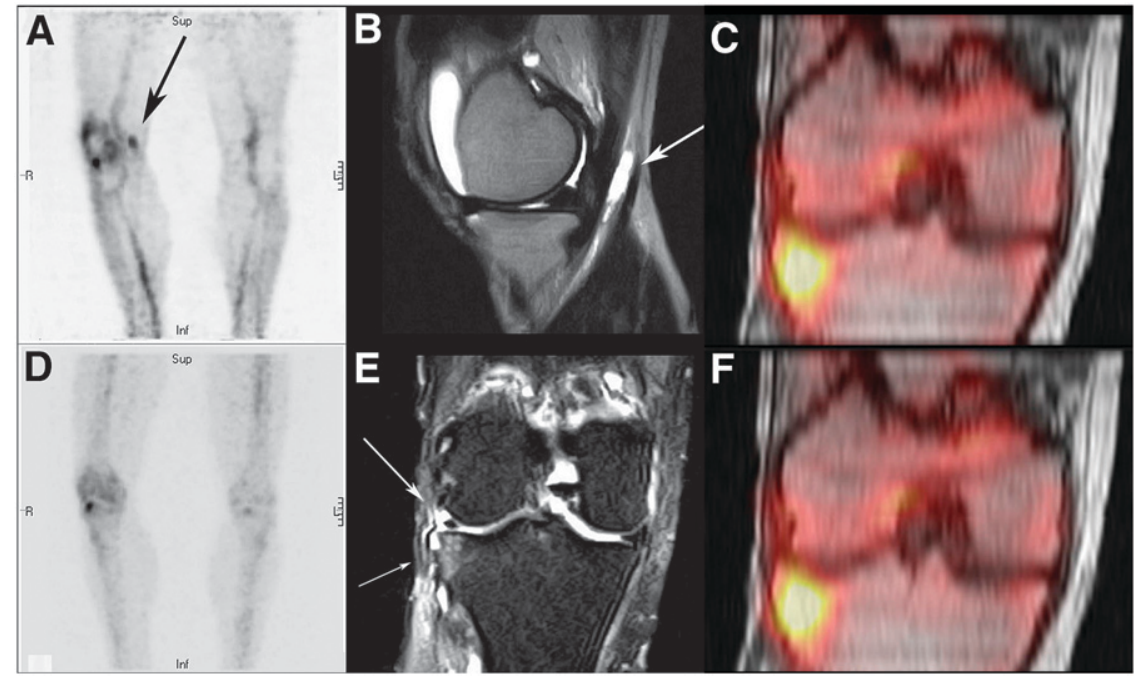

FIGURE 10. Lateral knee pain with previous lateral meniscectomy. (A) Blood-pool scan showing hyperemia of synovial lining (pes anserine bursitis) and bony lateral tibial plateau. Arrow points to bursitis. (B) Large joint effusion and anserine bursitis (arrow) in same medial area of knee as in A. (D) Delayed image showing only single remaining hot area for lateral plateau. (E) Coronal short- $\tau$ inversion recovery $\mathrm{MR}$ image showing intact meniscal remnant (large arrow) and lateral tibial plateau signal abnormality (small arrow). (C and F) Fused ${ }^{18} \mathrm{~F}$ scan and MR image of knee localizing and confirming lateral plateau injury.

fracture) in the vertebral body, shown on the accompanying 3 -axis and MIP imaging. The focus of uptake was more sharply defined and smaller than the increased T2-weighted MRI signal, further confirming bone edema.

\section{Case E}

Case E, shown in Figure 10, was a patient with persistent lateral knee pain after a partial lateral meniscectomy. The blood-pool study detected increased activity of the lateral tibial plateau and the synovial lining of the knee, and focal extraarticular soft-tissue uptake. This extraarticular softtissue activity corresponded to pes anserine bursitis near the semimembranosis tendon. The coronal short- $\tau$ inversion recovery image showed signal changes to the lateral plateau and the residual lateral meniscus. Fused T1-weighted coronal MRI and ${ }^{18} \mathrm{~F}$ PET bone scanning localized delayed bone scan activity to the lateral tibial plateau. Such uptake may be seen with meniscal dysfunction. As such, the patient's symptoms may include not only bone pain but also inflammation from the joint synovitis and the poplitial cyst.

\section{Case F}

Case F, shown in Figure 11, was a 43-y-old woman who experienced subtle pain behind the knee when climbing the stairs. Radiography of the knee revealed a highly sclerotic endosteal and marrow lesion. Reformatted coronal and

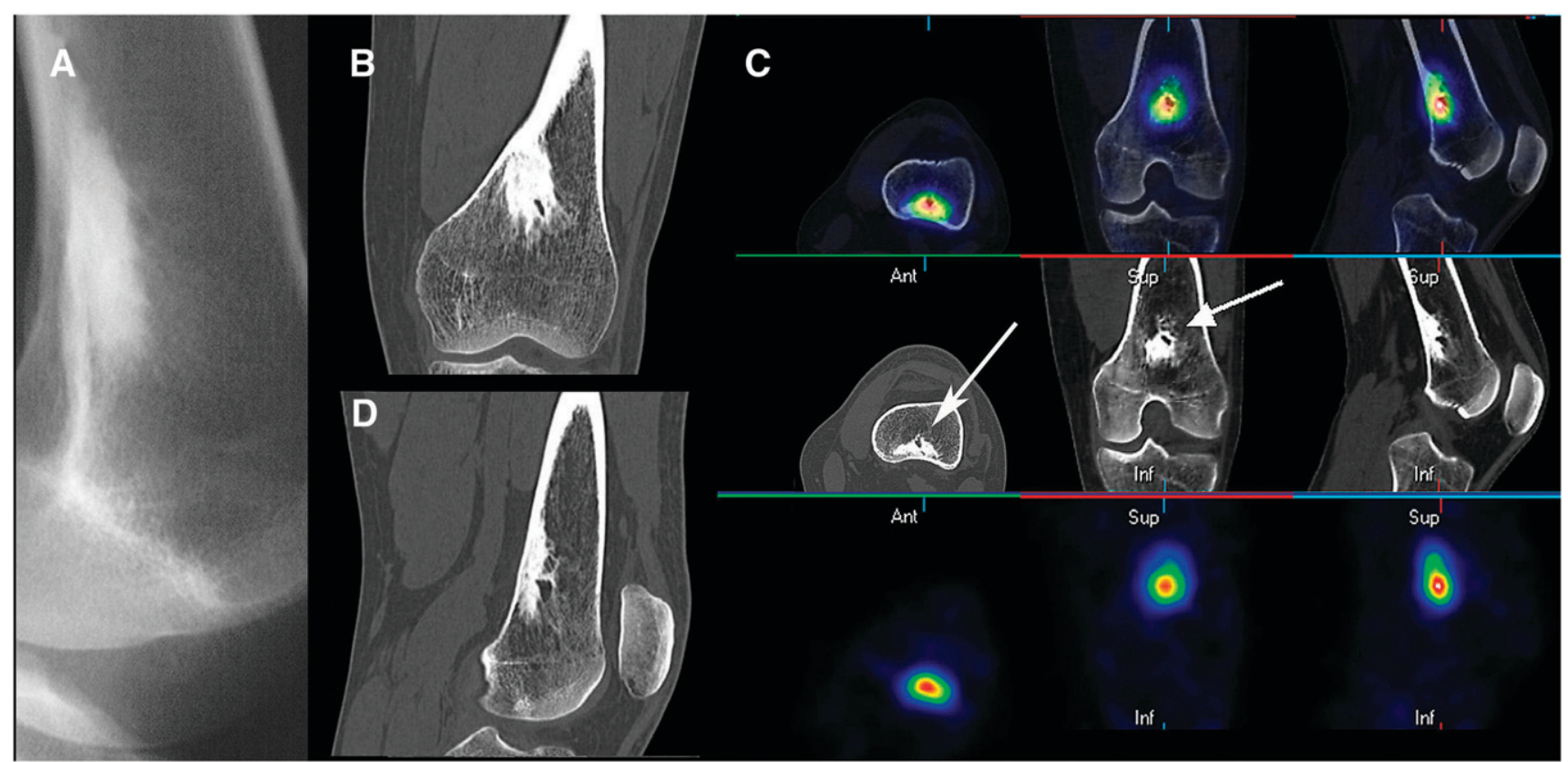

FIGURE 11. Deep knee pain in young woman: osteoid osteoma. (A) Radiograph of irregular, highly sclerotic endosteal/trabecular lesion of distal femur. (B and D) Coronal and sagittal CT slices of highly sclerotic lesion with cystic internal margin. (C) Three-axis montage of fused PET bone/CT, CT, and PET bone scan of lesion (arrows) showing greatest uptake at margin of cystic area. 
sagittal CT images showed the highly sclerotic area to be broadly based at the endosteum and peaking at a lucent nidus deep in the marrow. In the montage of images of the fused CT and PET bone scan, the greatest activity occurred adjacent to the nidus and decreased toward the endosteal margin. The area now has been stable for $2 \mathrm{y}$ and is considered to be an osteoid osteoma. Initial clinical response to the radiographic interpretation almost led to an immediate bone biopsy. Benign reactive bone may be misleading on pathologic review, appearing similar to and potentially being confused with a bone malignancy. Fusion can often redirect the diagnosis.

\section{Case G}

Case G, shown in Figure 12, was a 5-y-old child with pain and limping of the left leg and normal radiographic findings. To shorten the early PET bone scan in this patient, only the emission images were obtained. Dose was reduced to be commensurate with weight. Blood-pool imaging, including anterior, lateral, and MIP images, showed an elongated area of increased activity involving the medial surface of the distal femur. Delayed imaging showed persistence of the activity in the cortex of the medial aspect of the distal femur. Subsequent MRI was followed by open biopsy, which discovered a sterile abscess adjacent to the femur. This case showed that pediatric scanning is also practical.

\section{Case H}

Case H, shown in Figure 13, was a man who incurred minimal trauma to the left foot and ankle with progression into a profound case of osteopenia and hyperemia characteristic of reflex sympathetic disease. The patient was on crutches with minimal weight bearing before the onset of new pain. Reformatted coronal slices showed bone loss to be severe, when portions of the normal right foot were compared with the affected left foot and ankle. The patient had persistent pain despite ankle and foot support. The PET bone scan demonstrated a generalized increase in uptake for the left ankle and foot, with several areas of increased uptake. However, fusion amplified the significance of the findings in 2 places. The ankle joint was mostly spared, but there was significant activity in 2 areas, the subtalar joint and the inferior aspect of the medial cuneiform-first metatarsal joint. Reexamination of the CT scan showed subtle cortical insufficiency fractures of both these areas. A cast was placed on the foot. The extent of bone disease can be so great that important but subtle changes may be overlooked. Again, fusion draws one back to the areas of abnormality for further assessment. The combining of these modalities (CT and PET) gives literally ghostlike images of nuclear medicine flesh and bone. This case showed that image fusion can play a significant role in difficult cases.

The final series of cases exemplifies the power of PET bone scanning and fusion with both CT and MRI for the challenge of back pain. In the mid-1980s, the late Dr. Phil Braunstein and I authored a paper on SPECT bone scanning in the diagnosis of back pain (22). Additional articles have

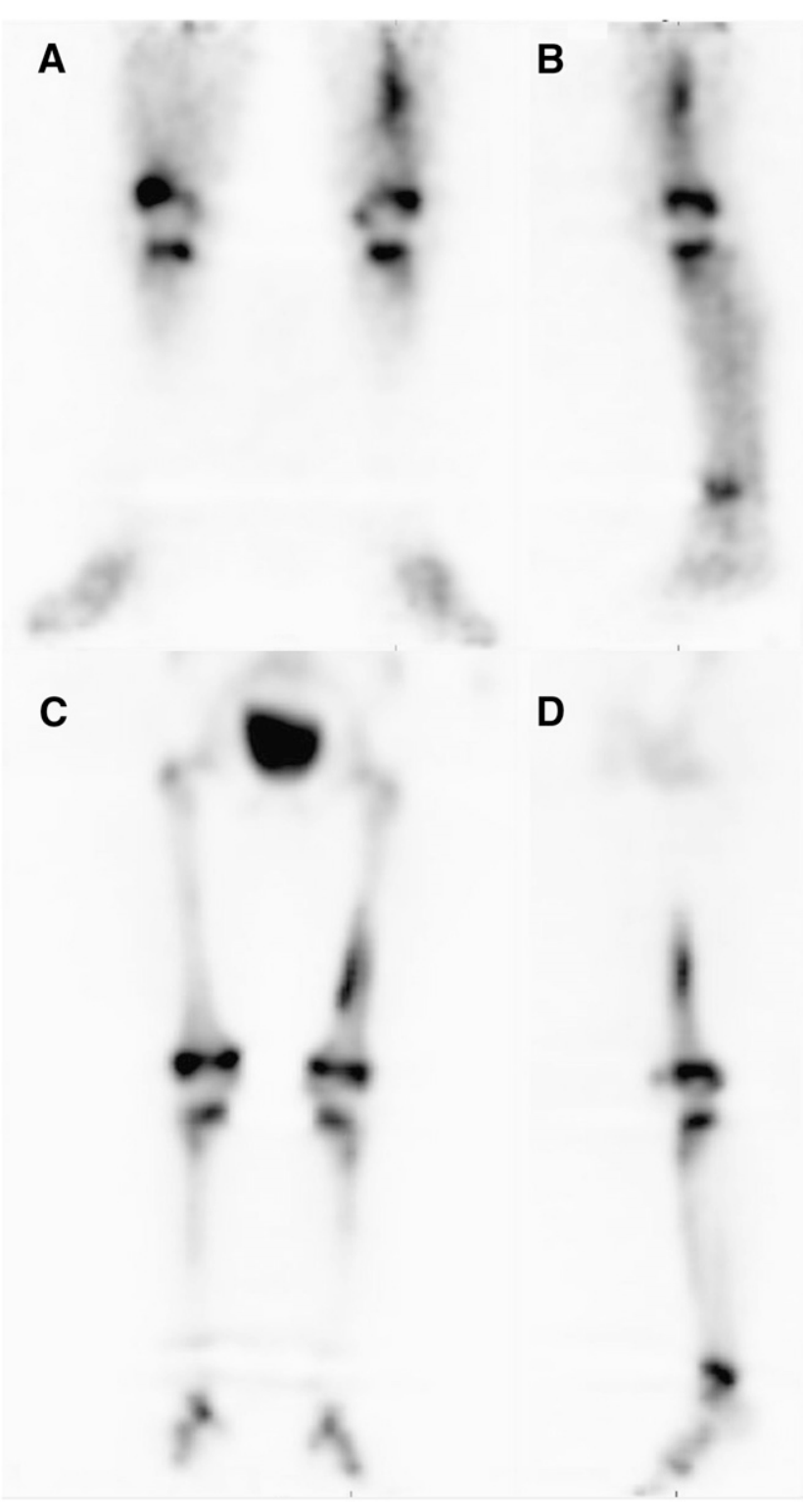

FIGURE 12. Dual-phase scan of young child with left leg limp and negative radiography findings. ( $A$ and $B$ ) Blood-pool scan positive for medial margin of distal shaft of left femur on anterior, lateral, and MIP images. (C and D) Delayed images continuing to be positive for superficial medial aspect of femur on similar views.

been published over the years on the use of SPECT. PET bone scanning elevates the diagnosis to a new level $(23,24)$.

\section{Case I}

Case I, shown in Figure 14, was a patient with a history of breast cancer, L4-L5 fusion for subluxation, and a new onset of back pain. The clinical concern was that the breast cancer might have metastasized or that fusion breakdown might have caused the back to fail. A montage of the sagittal T2-weighted images (left lateral margin, central, and right lateral margin) showed subluxation of L4 on L5 but without abnormal activity for the endplates. Both scans 


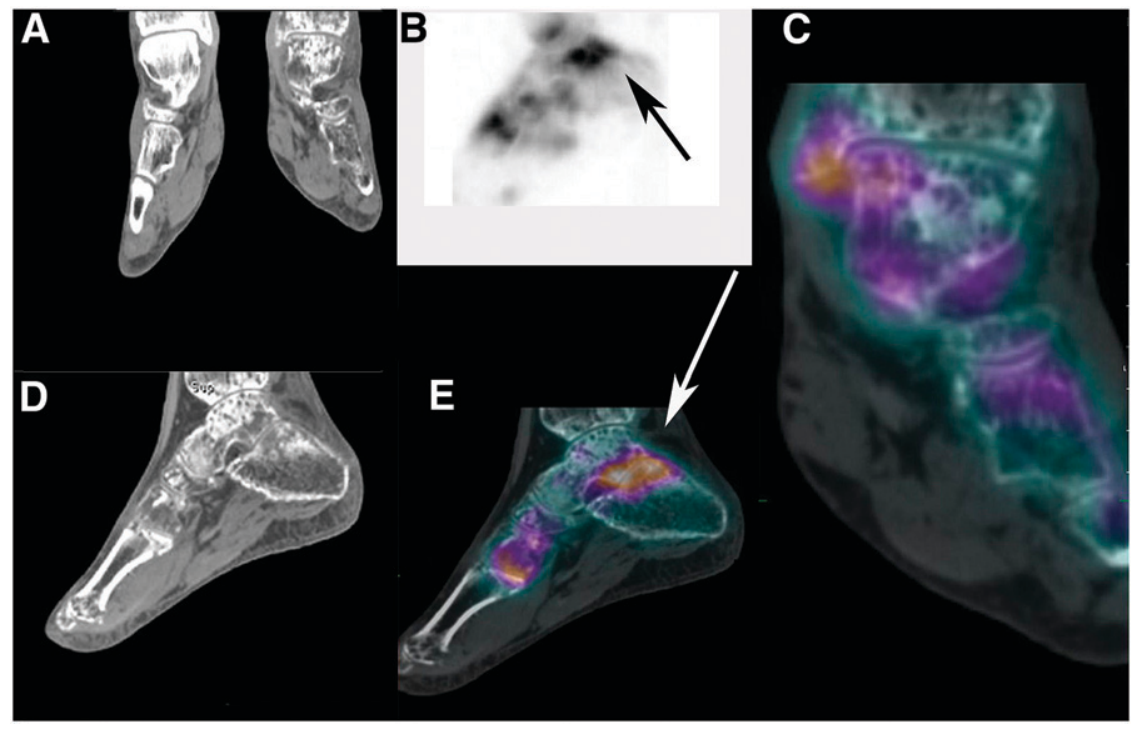

FIGURE 13. New onset of pain in left foot in patient with reflex sympathetic dystrophy. (A and D) Reformatted coronal slice through both ankles and sagittal slice of left ankle and foot showing profound demineralization of left ankle and foot. (B) Lateral MIP of ${ }^{18} \mathrm{~F}$ PET scan of left ankle with increased uptake (arrow) in subtalar joint. (C) CT and ${ }^{18} \mathrm{~F}$ fusion of normal uptake in ankle joint with minimal increase in uptake for medial malleolus. (E) Sagittal image of CT and ${ }^{18} \mathrm{~F}$ fusion showing abnormal uptake (arrow) in subtalar joint and first MTP joint. Close examination of CT images showed subtle insufficiency fractures for both areas. Fusion redirected attention to those areas where subtle insufficiency fractures were found on CT. Ankle joint is spared, as seen in rightmost fused coronal image.

excluded bony metastases with normal marrow activity. The fused MRI/PET bone scan further clarified the issue. Surgical fusions were intact. Although no malignant disease was present on the MRI and PET bone scan, the left facet joint superior to the fusion was actively degenerative and positive for uptake on both the bone scan and MRI fusion as seen on transaxial and sagittal images (arrows). Fusion further defined the activity as limited to the superior facet area rather than the immediately inferior facet fusion site. A diagnostic facet block could be beneficial and also might be therapeutic. Fusion also showed no abnormal activity for the arachnoid cyst at the right S2 level.

\section{Case J}

Case J, shown in Figure 15, was an elderly man who presented with a history of back pain and deep pelvic pain. Although there were obvious lumbar compression fractures, especially for L1, the somewhat diffuse uptake in the sacrum on the PET bone scan, the PET scan, and CT fusion become diagnostically significant after "connecting the dots" of activity extending from sacral foramina to foramina across the sacrum. A buckling of the anterior wall of S3 was seen on the sagittal fusion image. This insufficiency fracture might have been causing the deep pelvic pain. The fusion of anatomic information and metabolic information, as exemplified in the current PET/CT hybrid literature, was demonstrated in this case because both modalities carried part but not all of the diagnostic information, whereas together with fusion, a more complete evaluation could be performed.

\section{Case K}

Case K, shown in Figure 16, was the extremely problematic case of a patient with a history of multiple compression fractures, several of which had been treated with cement kyphoplasty. He returned with new back pain and a provisional diagnosis of a new L1 compression fracture on outside MRI. The 3D CT images showed kyphoscoliosis with multiple compression fractures. The sagittal 3D image was cut down the middle to show the numerous white cement kyphoplasties and sclerotic compressed vertebrae. Windowing the PET bone scan component of the fusion to reflect only the most intense areas of increased uptake allowed identification of several endplate compression fractures, including the superior endplate of L1 and the mid thoracic and lower lumbar levels. An asymptomatic right rib fracture was also seen. A lower thoracic kyphosis resulted in increased degeneration of facets, which might

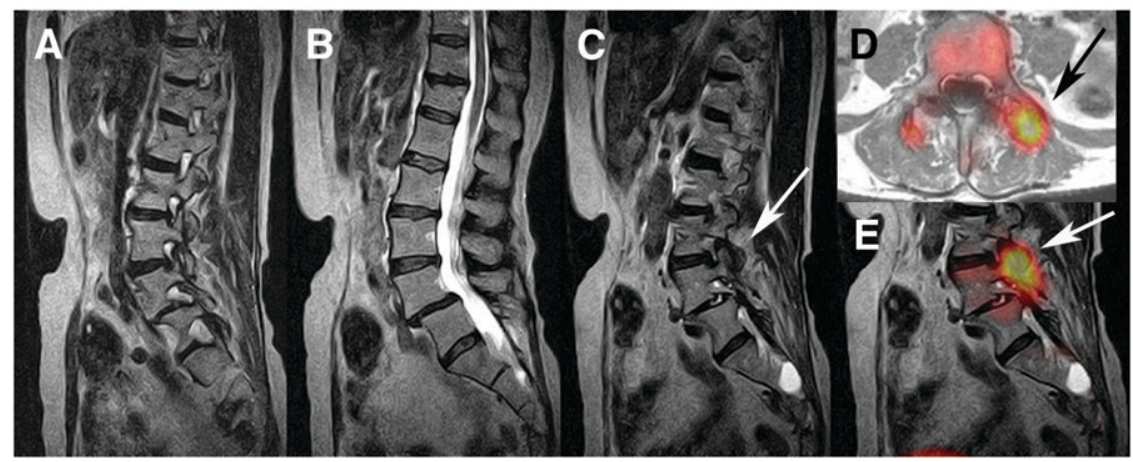

FIGURE 14. New-onset back pain in breast cancer patient with previous L4L5 fusion. (A-C) Sagittal slices of T2weighted MRI of lumbar spine without evidence of metastases. L4-L5 posterior fusion and facets are unremarkable on MRI. (C, D, and E) MRI, ${ }^{18} \mathrm{~F}$, and MRI fusion showing, on transaxial slice, marked uptake in left L3-L4 facet from progressive arthritis (arrows). Combined imaging excluded malignancy. Directed facet block could be both diagnostic and therapeutic. 
FIGURE 15. Increasing back pain and deep pelvic discomfort in elderly man. (A-C) Upper frames of $\mathrm{CT}$ and ${ }^{18} \mathrm{~F}$ fusion showing increased activity across sacrum and compression fractures of $L 1, L 2, L 4$, and L5. Note buckle fracture of anterior wall of S3 (arrow). (D and E) Lower frames of delayed scan showing numerous areas of increased uptake in lumbar spine but ill-defined cluster of activity across sacrum. (F) MIP of ${ }^{18} \mathrm{~F}$ scan. "Connecting the dots" in sacrum identifies early sacral insufficiency fracture.

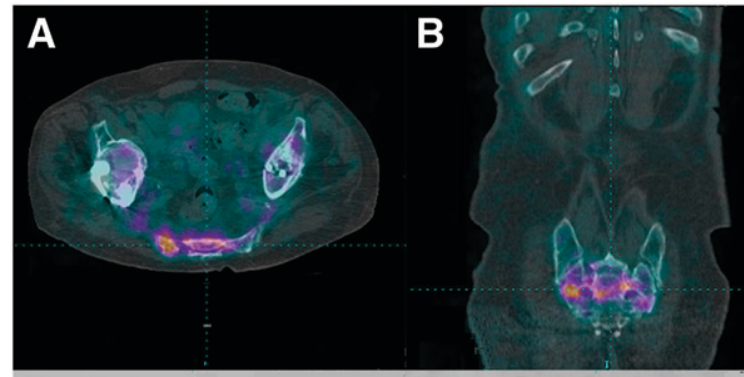

C

D

E

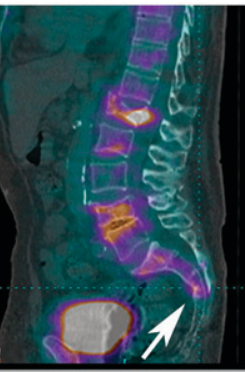

$\mathbf{F}$ also have been symptomatic. Endplate degenerative joint disease was also seen. Close examination of the areas of cement kyphoplasty showed that the remaining portions of the vertebral bodies, buttressed by the cement, had normal activity. This finding might have indicated that the patient's kyphoplasties stabilized the vertebral bodies and that the patient could potentially heal future compression fractures with additional cement support. Fusion imaging allowed a more accurate inventory and assessment of the numerous areas of disease.

\section{Case L}

Case L, the final case, is shown in Figure 17 and brings into focus the power of PET bone scanning with its high resolution. A 46-y-old woman underwent front-back lumbar spine fusion. In the immediate postoperative period, she slipped, felt "something give," and experienced persistent back pain thereafter. The CT sagittal reformatted image showed that the interbody spacer at L4-L5 had slipped forward, extending beyond the margins of the vertebral bodies. From the transaxial CT images, one could see that there were 2 channels in these cassettes for placing bone chips for osseous fusion. At the L4-L5 level, the channels were less dense and there was a small nonsupportive spiculation of bone lying outside the left posterior lateral margin of the L4-L5 cassette. With fusion, which benefits from the spatial and contrast resolution of ${ }^{18} \mathrm{~F}$ bone scanning and the CT, we could interrogate each channel for bony integrity. The fused images showed that the bone grafts at L4-L5 failed, with no activity (viable bone) seen to be crossing the disk space (small white arrows) through the channels, whereas the grafts were fully intact at L5-S1. Only mechanical compression by the spine and anterior longitudinal ligament held the L4-L5 cassette in place.

\section{DISCUSSION}

Many physicians are interested in starting PET bone scanning but are concerned about cost, appropriate coding, and the likelihood of successful reimbursement. At present, there are no specifically defined current procedural terminology

FIGURE 16. New-onset back pain with numerous compression fractures and kyphoplasties. (A) 3D CT of spine and pelvis showing numerous compression fractures. (B) Midsagittal slice through 3D column showing kyphoplasties and fractures. (C) ${ }^{18} \mathrm{~F}$ and $\mathrm{CT}$ fusion image showing several compression fractures, including new L1 superior endplate fracture and right rib fracture. (D) Fusion of ${ }^{18} \mathrm{~F}$ scan with $\mathrm{CT}$ scan showing readily identifiable correlation of vertebral bodies and areas of disease, including localization of compression fractures and facet

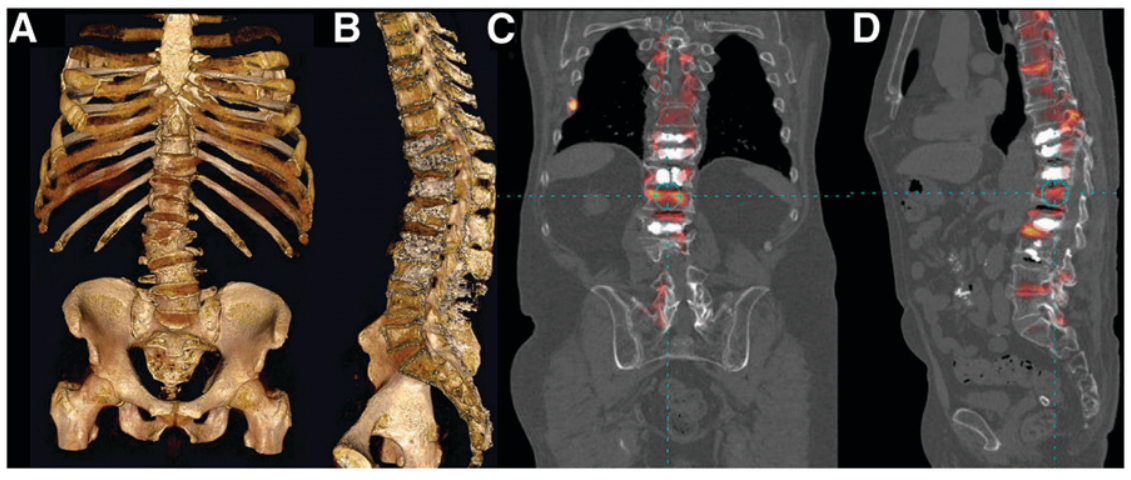
disease. Normal activity around kyphoplasties (chalky white interiors of several vertebral bodies) may indicate adequate stabilization after cementing. 


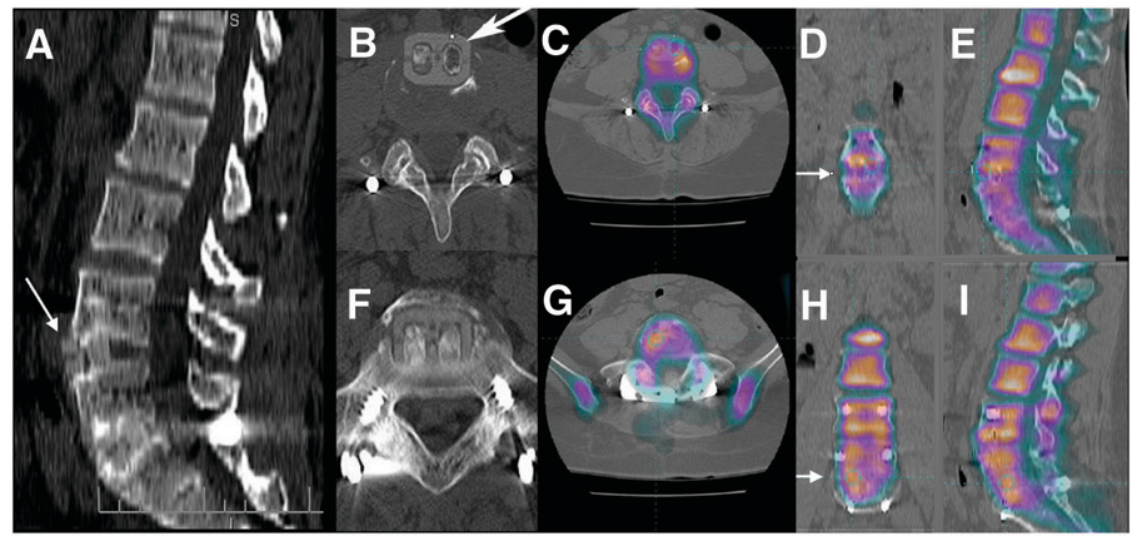

FIGURE 17. Failed front-back lumbar fusion: patient fell after recent surgery. (A) CT sagittal reformatted image shows that L4-L5 anterior interbody cassette has subluxed forward (arrow). L5-S1 cassette is in place. ( $B$ and $C$ ) Transaxial CT image of slipped cassette at L4-L5 (arrow) and fused PET bone/CT image of area of devitalized bone. ( $\mathrm{D}$ and $\mathrm{E}$ ) ${ }^{18} \mathrm{~F}$ and CT fusion in coronal and sagittal planes confirms devitalization of bone grafts at L4-L5. No activity crosses space between L4 and L5 (arrow in D). ( $F$ and $G$ ) CT and CT fusion of L5-S1. (H and I) Images showing that vital bone links L5 and S1 (arrow in H), unlike absence of activity across endplates at L4-L5. Improved resolution of ${ }^{18} \mathrm{~F}$ PET bone scanning allows interrogation of separate channels of cassettes.

(CPT) billing codes for PET bone scanning - neither for standalone PET nor for PET/CT. There is no CPT code for computer fusion of PET scans with CT or MRI scans. Reimbursement has not been consistent across third-party payers. One may choose to bill under an unspecified PET CPT code. One cannot use a SPECT code because PET is dual-photon imaging.

We have chosen to bill the study as a basic bone scan, with a modicum of success. The PET scanner, although sophisticated, is a $\gamma$-camera. The tracer has been twice approved by the Food and Drug Administration, once for general bone scintigraphy and again later for PET. The technologic strides that have combined PET with computerized x-ray tomography for attenuation-corrected and coregistered imaging have improved diagnosis, but with an added complexity and cost that require appropriate reimbursement. Still, the elevation of an imaging modality from about $80 \%$ to nearly $100 \%$ sensitivity and specificity is important clinically.

Taking a simplistic but proactive objective approach to the value of a PET bone scan may be beneficial for discussion at this early stage. The cost of new technology is nonlinear. The 25\% improvement in sensitivity and specificity of PET bone over planar bone scans required far more than a $25 \%$ increase in cost with the replacement of planar imaging by computerized tomographic scanning. The 3 components for the cost of a PET bone scan are professional, technical, and isotopic charges. The interpretation is not as technically challenging for a PET bone scan as for ${ }^{18} \mathrm{~F}$-FDG PET, but there are more areas to evaluate than on planar bone or single SPECT study. Professional reimbursement should fall between the values for ${ }^{18} \mathrm{~F}$-FDG and SPECT. Unlike ${ }^{18} \mathrm{~F}$-FDG PET, which provides a completely new imaging modality, the ${ }^{18} \mathrm{~F}$ bone scan is not unique, replacing a similar and well-established study by providing better diagnostic clarity. The only direct comparison for the technical component is SPECT. Using coverage as a model, the whole-body ${ }^{18} \mathrm{~F}$ PET scan is equal to the at least 4 or 5 SPECT scans needed to cover the same areas from head to toe. Scans of the trunk would have a higher likelihood of showing disease than would scans of the more inferior parts of the lower extremities. Thus, a projected value could be the value of a base planar bone scan plus 3-5 times the incremental increase in the cost of a SPECT study over planar imaging. The cost of the isotope will be higher than ${ }^{99 \mathrm{~m} T c}$-MDP but lower than ${ }^{18} \mathrm{~F}$-FDG. We presently pay 3 times the cost of ${ }^{99 \mathrm{~m}} \mathrm{Tc}-\mathrm{MDP}$ for each dose of $\mathrm{Na}^{18} \mathrm{~F}$. An additional reimbursement charge is needed for computer-based fusion and motion correction. This charge can be similar to the present charges for computerized 3D imaging as used in bone and vascular studies.

\section{CONCLUSION}

These case studies demonstrate the versatility of ${ }^{18} \mathrm{~F}$ bone scans for the same scope of practice as is covered by conventional ${ }^{99 \mathrm{~m}} \mathrm{Tc}-\mathrm{MDP}$ imaging. The increased sensitivity and specificity of PET bone scans, approaching 100\%, may provide a more thorough and conclusive evaluation of bone disease. PET bone scans can also provide an additional source of revenue for PET scanners in low-volume markets. PET bone scans are easy to do and lend themselves to multimodality fusion with CT and MRI. Used as a dual-tracer cocktail, combined ${ }^{18} \mathrm{~F}-\mathrm{FDG}$ and ${ }^{18} \mathrm{~F}$ can reduce the cost and time of diagnosis in certain diseases. ${ }^{18} \mathrm{~F}$ may become the "contaminate of choice" for the more esoteric tracers to ensure fusion alignment when there are no other anatomic markers to confirm location.

Implementation can be challenging, but only through implementation can PET bone scanning move into the present-day imaging arena and earn the reimbursement it is due. Once one performs a PET bone scan, there can never be satisfaction with reverting back to the "old" bone scans. The primary justification for presently performing PET bone scans centers directly on improved patient care and, it is hoped, improved patient outcomes.

The circle is now complete. An old isotope is now the new isotope. The curtain rises on a new act. 


\section{ACKNOWLEDGMENTS}

As first author for this article, I acknowledge the teamwork that made this article possible. The successful implementation of new imaging protocols and fusion techniques required an integration of skills and training exemplary of the level of training and experience of the team's technologists. The early technical contributions provided by Seattle Nuclear have been greatly appreciated. I also acknowledge those who have been mentors and colleagues. I greatly appreciate the contributions of my mentors Dr. Richard Friedenberg, who was the chairman of my radiology residency at UC-Irvine, and the late Dr. Phillip Braunstein, head of Nuclear Medicine at UC-Irvine during my residency and fellowship and colleague during my time there as an assistant professor. Presently, I acknowledge Dr. Alan Waxman for his help, instruction, guidance, and insight into the challenging arena of PET imaging.

\section{REFERENCES}

1. Schirrmeister H, Guhlmann A, Kotzerke J. Early detection and accurate description of extent of metastatic bone disease in breast cancer with fluorine ion and positron emission tomography. J Clin Oncol. 1999;17:2381-2389.

2. Einat Even-Spair, Ur Metser, Eyal Mishani, Gennady Lievshitz, Hedva Lerman, Ilan Leibovitch. The detection of bone metastases in patients with high-risk prostate cancer: 99mTc-MDP planar bone scintigraphy, single and multi-field-of-view SPECT, ${ }^{18}$ F-fluoride PET and ${ }^{18}$ F-fluoride PET/CT. J Nucl Med. 2006;47:287-297.

3. Blahd WH. Ben Cassen and the development of the rectilinear scanner. Semin Nucl Med. 1996;26:165-170.

4. Spencer R, Herbert R, Rish WM, et al. Bone scanning with $85-\mathrm{Sr}, 87 \mathrm{~m}-\mathrm{Sr}$ and 18-F: physical and radiopharmaceutical considerations and clinical experience in 50 cases. Br J Radiol. 1967;40:641-654.

5. Nutt R. The history of positron emission tomography. Mol Imaging Biol. 2002;4:11-26.

6. Tucker D, Greene MW, Weiss AJ, Murrenhoff A. Methods of Preparation of Some Carrier-Free Radioisotopes Involving Sorption on Alumina. Upton, NY: Brookhaven National Laboratory; 1958. Report no. BNL-3746.

7. Subramanian G, McAfee JG. A new complex of Tc-99m for skeletal imaging. Radiology. 1971;99:192-196.

8. Tapscott E. First scintillation camera is foundation for modern imaging systems. J Nucl Med. 1998;39(3):15N-27N.
9. Positron Emission Tomography Drug Products: Safety and Effectiveness of Certain PET Drugs for Specific Indications [Food and Drug Administration Web site]. Available at: www.fda.gov/ohrms/dockets/98fr/031000a.txt. Accessed March 19, 2007.

10. Messa C, Goodman WG, Hoh CK, et al. Bone metabolic activity measured with positron emission tomography and $\left[{ }^{18} \mathrm{~F}\right]$ fluoride ion in renal osteodystrophy: correlation with bone histomorphometry. J Clin Endocrinol Metab. 1993;77: 949-955.

11. Hoh CK, Hawkins RA, Dahbom M, et al. Whole body skeletal imaging with $\left[{ }^{18}\right.$ F]fluoride ion and PET. J Comput Assist Tomogr. 1993;17:34-41.

12. Attachment II: Sample Formats-Labeling for Ammonia N 13 Injection, Fludeoxyglucose F 18 Injection $\left[{ }^{18}\right.$ F] FDG, and Sodium Fluoride F 18 Injection [Food and Drug Administration Web site]. Available at: www.fda.gov/cder/ guidance/labsample.pdf. Accessed March 19, 2007.

13. Tarantola G, Zito F, Gerundini P. PET instrumentation and reconstruction algorithms in whole-body applications. J Nucl Med. 2003;44:756-796.

14. Levin CS, Hoffman EJ. Calculation of positron range and its effect on the fundamental limit of positron emission tomography system spatial resolution. Phys Med Biol. 1999;44:781-799.

15. Even-Sapir E. Imaging of malignant bone involvement by morphologic, scintigraphic, and hybrid modalities. J Nucl Med. 2005;46:1356-1367.

16. Schirrmeister H, Guhlmann A, Elsner K, et al. Sensitivity in detecting osseous lesions depends on anatomic localization: planar bone scintigraphy versus ${ }^{18} \mathrm{~F}$ PET. J Nucl Med. 1999;40:1623-1629.

17. Weber DA, Makler PT Jr, Watson EE, Coffey JL, Thomas SR, London J. Radiation absorbed dose from technetium-99m-labeled bone imaging agents. Task Group of the Medical Internal Radiation Dose Committee, the Society of Nuclear Medicine. J Nucl Med. 1989;30:1117-11.

18. Mawlawi O, Podoloff DA, Kohlmyer S, et al. Performance characteristics of a newly developed PET/CT scanner using NEMA standards in 2D and 3D modes. J Nucl Med. 2004;45:1734-1742.

19. Mullani NA, Mawlawi O. Why is the resolution of the discovery PET/CT camera so poor? [letter]. J Nucl Med. 2005;46:898.

20. Lee J, Park CH, Kim HC, Kim HS. Dichotomy between Tc-99m MDP bone scan and fluorine-18 fluorodeoxyglucose coincidence detection positron emission tomography in patients with non-Hodgkin's lymphoma. Clin Nucl Med. 2000;25: 532-535.

21. Wade AA, Scott JA, Kuter I, Fischman AJ. Flare response in ${ }^{18}$ F-fluoride ion PET bone scanning. AJR. 2006;186:1783-1786.

22. Braunstein P, Bridges RL. Single-photon emission computed tomography bone imaging: a new dimension in the investigation of back pain. Epitome-Nuclear Medicine West J Med. 1986;144:214.

23. Pneumaticos SG, Chatziioannou SN, Hipp JA, Moore WH, Esses SI. Low back pain: prediction of short-term outcome of facet joint injection with bone scintigraphy. Radiology. 2006;238:693-698.

24. Holder LE, Machin JL, Asdourian PL, Links JM, Sexton CC. Planar and high resolution SPECT bone imaging in the diagnosis of facet syndrome. J Nucl Med. 1995;36:37-44. 